\title{
BIOPSIE ET MESURE DE L'ACTIVITÉ NA+/K+ ATPASIQUE BRANCHIALE : VALIDITÉ ET IMPACT SUR LE DÉVELOPPEMENT DU SMOLT DE SAUMON ATLANTIQUE (SALMO SALAR L.).
}

\author{
L. SIEGLER (1, 2), H. D'COTTA (3), L. PAULIN (2), J.L. BAGLINIÈRE (2), P. PRUNET (3)
}

(1) Ecole Nationale Supérieure Agronomique de Rennes, UFR Ecologie et Sciences Phytosanitaires, Ecologie des Hydrosystèmes Continentaux, 65 rue de St Brieuc, 35042 Rennes Cedex, France.

(2) Institut National de la Recherche Agronomique, Laboratoire d'Ecologie Aquatique, 65 rue de St Brieuc, 35042 Rennes Cedex, France.

(3) Institut National de la Recherche Agronomique, Laboratoire de Physiologie du Poisson, Campus de Beaulieu, 35000 Rennes, France.

\section{RÉSUMÉ}

Une biopsie de fragment de tissu branchial a été effectuée, durant toute la période de smoltification, sur des juvéniles de saumons Atlantique (Salmo salar) élevés en pisciculture. Le microdosage de l'activité de la Na+/K+ ATPase branchiale, effectué sur ce fragment de tissu, a été comparé au macrodosage obtenu à partir des branchies entières. Aucune mortalité n'a été observée chez les saumons biopsiés dont le taux de croissance (linéaire et pondérale) est resté identique à celui des poissons témoins. La bonne correspondance des cinétiques de l'activité enzymatique obtenues par les deux méthodes valide la technique de microdosage et confirme l'absence d'influence sur le développement physiologique ultérieur du smolt biopsié. Nos résultats montrent les modifications envisageables pour améliorer la sensibilité de la méthode. Cette technique est très prometteuse pour l'analyse des populations naturelles.

Mots-clés : biopsie, NA+/K+ ATPase, branchie, Salmo salar.

\section{BIOPSY AND MEASUREMENT OF BRANCHIAL NA+/K+ ATPASE ACTIVITY : VALIDITY AND IMPACT ON ATLANTIC SALMON (SALMO SALAR L.) SMOLT DEVELOPMENT.}

\section{ABSTRACT}

A biopsy of a fragment of gill tissue was performed, during the whole smolting period, on juvenile hatchery reared Atlantic salmon (Salmo salar L.). The microassay of the $\mathrm{Na}+\mathrm{K}+$ ATPase activity was compared with the macroassay done on the totality of gill arches. No mortality was observed in the gill biopsy group whose growth rate (length and weight) was similar to the control group. The good correspondence of the kinetics on the enzyme activity validates the microassay method and confirms that the biopsy has no effect on the posterior physiologic development of smolts. Our results show the envisaging changes in the method to enhance the microassay sensitivity. The technique is very promising for the analysis of natural populations.

Key-words : biopsy, NA+/K+ ATPase, gill, Salmo salar. 


\section{INTRODUCTION}

Chez les salmonidés, l'augmentation de l'activité de l'Adenosine Tri-Phosphatase Sodium-Potassium branchiale au cours de la transformation pari-smolt en fait un bon indicateur du niveau de smoltification et donc un bon indicateur de l'adaptation du poisson au milieu marin (FOLMAR et DICKHOFF, 1980 ; BOEUF et al., 1982 ; MicCORMICK et al., 1985 ; SOIVIO et al., 1989 ; BOEUF, 1992 ; ROPARS, 1993 ; TANGUY, 1993).

Jusqu'à présent, la mesure de l'activité de la $\mathrm{Na}+\mathrm{K}+$ ATPase branchiale demandait des quantités de tissus telles qu'elle nécessitait de sacrifier le poisson (LASSER et al., 1978 ; STAGG et SHUTTLEWORTH, 1982 ; ZAUGG,1982). Lors des études en milieu naturel, ce sacrifice systématique induit une modification artificielle des effectifs et du taux de survie dont les effets sur la dynamique de population sont difficiles à estimer.

MCCORMICK (1993) et SCHROCK et al. (1994) ont développé respectivement sur saumons Atlantique (Salmo salar) et sur saumons du Pacifique (Oncorhynchus spp) des techniques de dosage fin (microdosage) de l'activité de la $\mathrm{Na}+\mathrm{K}+$ ATPase ne nécessitant qu'une faible quantité de tissu branchial. Ce fragment de tissu peut être prélevé par biopsie en minimisant de ce fait les traumatismes occasionnés au poisson.

L'étude présentée a pour but de comparer les résultats obtenus par_cette méthode (microdosage) à ceux observés par l'analyse des branchies entières (macrodosage), non plus ponctuellement, mais sur une période de temps continue (4 mois). Dans ce cadre, elle permet également de vérifier à moyen terme l'absence d'impact de la biopsie sur la survie mais également sur la croissance tant linéaire que pondérale ainsi que sur le développement physiologique du jeune salmonidé. II convenait également d'évaluer la fiabilité des résultats obtenus afin de déterminer les possibilités d'application d'une telle méthode pour des études en routine, notamment sur des populations de truites fario (Salmo trutta) et de pouvoir proposer, le cas échéant, quelques améliorations.

Le test de cette nouvelle méthode a été effectué en milieu contrôlé, sur une population de saumons atlantiques (Salmo salar) pendant toute la période de smoltification (mars à juin).

\section{MATÉRIEL ET MÉTHODES}

\section{Récoltes des données en pisciculture}

\section{Les poissons}

La méthode de biopsie a été testée sur des juvéniles de saumon atlantique (Salmo salar) élevés à la pisciculture expérimentale du Drennec, cogérée par l'INRA et l'IFREMER. Les poissons étaient maintenus dans des bacs de plein air alimentés continuellement en eau courante à partir de l'eau de la rivière voisine, l'Elorn.

Les saumons étaient prélevés de façon aléatoire dans un bac ( $A$ ) contenant des juvéniles issus d'une même cohorte et identifiés comme pré-smolts ou-smolts au début de mars d'après leur phénotype (robe argentée, disparition des marques de digitation latérales, apparition de stries noires sur la nageoire caudale). Les poissons non prélevés constituaient le lot témoin. Les smolts biopsiés étaient placés dans un bac séparé $(B)$ pour contrôler leur survie, leur croissance et leur état général.

\section{Protocole et manipulation des poissons}

Sept campagnes de mesures ont été mises en place entre le début du mois de mars et la mi-juin, de manière à couvrir toute la période de smoltification. Les prélèvements étaient espacés de quinze jours, sauf le dernier, espacé d'un mois.

Les poissons étaient manipulés sous anesthésie (Phénoxy-éthanol $3 \mathrm{ml} / \mathrm{l}$ ), sur des supports humides. Durant les différentes manipulations, ils étaient stockés dans une eau identique à celle des bacs d'élevage.

A chaque campagne de mesures, vingt-quatre individus dont l'avancement de la smoltification a été évalué selon les critères phénotypiques précédemment décrits, ont été 
mesurés (longueur fourche et totale) et pesés. Douze ont été biopsiés au milieu du premier arc branchial gauche et mis en bac B. Les branchies des douze autres ont été complètement prélevées pour effectuer une mesure de l'activité $\mathrm{Na}+, \mathrm{K}+$, ATPasique par macrodosage. Puis, les poissons biopsiés lors des campagnes précédentes ont été contrôlés (nombre, longueur fourche, longueur totale, poids, état sanitaire et avancement de la cicatrisation) et comparés à un échantillon aléatoire de cinquante témoins non traités pour pouvoir déceler d'éventuelles différences de croissance.

Lors de la dernière campagne de mesures, des prélèvements supplémentaires ont été réalisés de la manière suivante. Sur un smolt, une biopsie a été réalisée au milieu de chaque arc branchial (8 prélèvements) ; ensuite, douze smolts ont été biopsiés puis la totalité de leur tissu branchial a été prélevée pour effectuer un macrodosage de l'activité ATPasique, enfin; douze smolts manipulés au cours des campagnes précédentes ont été pris au hasard dans le bac $B$ et biopsiés une seconde fois.

\section{Prélèvement du tissu branchial}

Pour la biopsie, l'opercule était maintenu écarté à l'aide d'une pince à bouts ronds, qui servait également à soulever le premier arc. Un échantillon de 4 à 6 filaments était prélevé avec une paire de petits ciseaux chirurgicaux à pointes recourbées. Pour éviter une hémorragie importante, la coupure était faite au-dessus du septum cartilagineux qui maintient les filaments soudés sur la moitié de leur longueur (cas des salmonidés et de certaines autres espèces).

Les filaments étaient ensuite immergés dans des tubes Eppendorfs de $1.5 \mathrm{ml}$ contenant $100 \mu \mathrm{l}$ de SEl (solution tampon : $0.3 \mathrm{~mol} / \mathrm{l}$ sucrose, $0.02 \mathrm{~mol} / \mathrm{l} \mathrm{Na}{ }_{2}$ EDTA, $0.1 \mathrm{~mol} / \mathrm{l}$ imidazole, $\mathrm{pH}$ 7.1) à la température de la glace.

Pour le macrodosage, la totalité des branchies était prélevée, rincée dans une solution de SEI pour éliminer le sang et placée dans des tubes Eppendorfs.

Les Eppendorfs étaient ensuite congelés instantanément dans l'azote liquide, puis stockés à $-80^{\circ} \mathrm{C}$.

\section{Analyses physiologiques}

\section{Analyse par macrodosage}

L'analyse des échantillons de branchies entières a été effectuée selon une technique de macrodosage dérivée de celle de ZAUGG (1982). L'activité $\mathrm{Na}+\mathrm{K}+\mathrm{KTP}$ asique est mesurée en dosant le phosphore inorganique libéré par hydrolyse de l'Adénosine triphosphate (ATP). Elle est mesurée en micromole de phosphate libéré par milligramme de protéine et par heure $\left(\mu \mathrm{mol} \mathrm{Pi} . \mathrm{mg}^{-1} . \mathrm{h}^{-1}\right)$. L'activité de cette enzyme est calculée par différence entre l'activité ATPasique totale et l'activité ATPasique en présence d'Ouabaïne (inhibiteur spécifique de la $\mathrm{Na}+/ \mathrm{K}+$ ATPase).

\section{Préparation des échantillons}

Les tubes Eppendorfs de $1.5 \mathrm{ml}$ contenant les arcs branchiaux ont été sortis du congélateur juste avant leur utilisation et ont été maintenus sur glace en permanence.

Les échantillons ont tout d'abord été homogénéisés par broyage des arcs branchiaux dans $1 \mathrm{ml}$ de SEI (Sucrose $0.3 \mathrm{M}, \mathrm{Na}_{2}$ EDTA $0.02 \mathrm{M}$, Imidazole $0.1 \mathrm{M}, \mathrm{PH} 7.1$ ) à l'aide d'un potter Weathon $7 \mathrm{ml}$. Les tissus ainsi séparés des parties cartilagineuses sont transvasés dans des tubes Eppendorfs de $2 \mathrm{ml}$ et dilués dans $1 \mathrm{ml}$ d'eau distillée.

Une première centrifugation de 7 minutes à $2000 \mathrm{~g}$ permet de recupérer les membranes cellulaires conservées dans le culot. Un broyage de ce culot dans 450 à $950 \mu \mathrm{l}$ de SEID (SEI + 1g/l de sodium déoxycholate) à l'aide du potter Weathon, permet la mise en solution des enzymes membranaires. Ces dernières sont récupérées dans le surnageant après une nouvelle centrifugation de 6 minutes à $2000 \mathrm{~g}$. 


\section{Protocole de dosage}

Le dosage du phosphate inorganique libéré a été réalisé selon une méthode dérivée de celle de ATKINSON et al. (1973) en duplicats dans des tubes de $5 \mathrm{ml}$ contenant d'une part $500 \mu \mathrm{l}$ de solution $\mathrm{A}\left(23 \mathrm{mmo} / / \mathrm{MgCl}, 6 \mathrm{H}_{2} \mathrm{O}, 155 \mathrm{mmol} / \mathrm{l} \mathrm{NaCl}, 75 \mathrm{mmol} / / \mathrm{KCl}, 115 \mathrm{mmol} / \mathrm{l}\right.$ imidazole, $\mathrm{pH}$ 7) pour la mesure de l'activité totale et d'autre part $500 \mu \mathrm{l}$ de solution A plus $0.58 \mathrm{mmoles} / \mathrm{l}$ d'ouabaïne (solution $\mathrm{B}$ ) pour la mesure de l'activité des ATPases non sensibles à l'ouabaïne. $20 \mu \mathrm{l}$ de préparation enzymatique puis $100 \mu \mathrm{l}$ d'une solution d'ATP (0.03 $\mathrm{M}$ de $\mathrm{Na}_{2}$ ATP, PH 7) ont été distribués dans chaque tube. Toutes ces opérations ont été effectuées dans un bain-marie froid $\left(0^{\circ} \mathrm{C}\right)$. Ensuite, les tubes ont été placés dans un bainmarie à $37^{\circ} \mathrm{C}$ pendant 10 minutes puis immédiatement refroidis dans la glace. $200 \mu \mathrm{l}$ d'eau distillée ont été ajoutés dans chaque tube, ainsi que $3 \mathrm{ml}$ de mélange lubrol-molybdate à $1 / 1$ (lubrol 17A17 : $10 \mathrm{~g} / \mathrm{l}$; ammonium heptamolybdate : 10g/l heptamolybdate, $100 \mathrm{ml} / / \mathrm{H}_{2} \mathrm{SO}_{4}$ ) pour stopper la réaction et fixer le phosphate inorganique libéré sous forme d'un complexe soluble jaune.

Simultanément à ces opérations, 2 blancs et 5 standards ont été préparés en duplicats dans des tubes de $5 \mathrm{ml}$ et soumis aux mêmes conditions. Les blancs contenaient $500 \mu \mathrm{l}$ de solution B, $20 \mu \mathrm{l}$ de SEID, $100 \mu \mathrm{l}$ de solution d'ATP, $200 \mu \mathrm{l}$ d'eau distillée et $3 \mathrm{ml}$ de mélange lubrol-molybdate à 1/1. Dans les standards, 20 à $100 \mu$ le solution de phosphate inorganique $(0.1 \mathrm{mg} \mathrm{Pi} / \mathrm{ml})$ ont été substitués au même volume d'eau distillée.

Après 20 minutes à $25^{\circ} \mathrm{C}$, les préparations ont été réparties sur une microplaque (96 puits) à raison de $300 \mu \mathrm{l}$ par puits. Le niveau de phosphate inorganique a alors été mesuré par spectrophotométrie à $405 \mathrm{~nm}$, sur un lecteur de micro-plaques ELISA (enzyme-linked immunosorbent assay).

Le dosage des protéines a été réalisé selon la méthode de BRADFORD (1976), dans des tubes de $10 \mathrm{ml}$, en duplicats. Chaque tube contenait $20 \mu \mathrm{l}$ de préparation enzymatique et $5 \mathrm{ml}$ de réactif (Biorad dye protein assay reagent) dilué à 1/4. Les 2 blancs (en duplicats) contenaient $20 \mu \mathrm{l}$ de SEID et $5 \mathrm{ml}$ de colorant dilué. Les 5 standards (en duplicats) contenaient en plus 20 à $100 \mu$ de BSA (Bovine Serum Albumine $1 \mathrm{mg} / \mathrm{ml}$ ). Les microplaques ont été remplies immédiatement de la manière précédente, et la lecture faite à $630 \mathrm{~nm}$.

\section{Analyse par microdosage}

La mesure de l'activité $\mathrm{Na}+/ \mathrm{K}+$ ATPasique sur les échantillons de biopsie de tissu branchial a été réalisée selon une méthode proche de celle proposée par McCORMICK (1993).

\section{Préparation des échantillons}

L'homogénéisation des échantillons et la mise en solution des enzymes membranaires ont été assurées par un unique broyage dans $25 \mu \mathrm{l}$ de SEID concentré $(5 \mathrm{~g} / \mathrm{l}$ sodium desoxycholate), préparé juste avant le dosage en raison de son importante viscosité. Un microbroyeur électrique (Kontes, Vineland, NJ, USA) a été utilisé, à raison de 1 minute par échantillon. Une seule centrifugation de 1 minute à $5000 \mathrm{~g}$ a été réalisée, le surnageant étant conservé dans le tube Eppendorf d'origine pour éviter les pertes.

\section{Protocole de dosage}

Le dosage du phosphate inorganique libéré par l'activité enzymatique a été réalisé selon le même principe que celui décrit précédemment, dans des tubes de $3 \mathrm{ml}$ en duplicats. Chacun contenait $200 \mu \mathrm{l}$ de solution A ou B, $15 \mu \mathrm{l}$ de préparation enzymatique, $40 \mu \mathrm{l}$ de solution d'ATP, $100 \mu \mathrm{l}$ d'eau distillée et $1.2 \mathrm{ml}$ de mélange lubrol-molybdate. Pour ce dosage, 9 standards et 2 blancs ont été préparés en duplicats. Dans les blancs et les standards, la préparation enzymatique a été remplacée par $15 \mu l$ de SEID. Dans les 9 standards, 5 à $60 \mu \mathrm{l}$ de solution de phosphate inorganique $(0.1 \mathrm{mg} \mathrm{Pi} / \mathrm{ml})$ étaient substitués au même volume d'eau distillée.

Le dosage des protéines était similaire à celui de la macrométhode. Les tubes $(3 \mathrm{ml})$ contenaient $15 \mu \mathrm{l}$ de préparation enzymatique, $800 \mu \mathrm{l}$ d'eau distillée et $200 \mu \mathrm{l}$ de réactif 
(biorad dye) non dilué. Dans les blancs et les standards, $15 \mu \mathrm{l}$ de SEID dilué remplaçaient la préparation enzymatique. Les standards étaient au nombre de 13 duplicats et contenaient de 1 à $40 \mu \mathrm{l}$ de BSA, le volume d'eau distillée ajouté étant diminué d'autant pour un même volume final.

\section{Traitement des données}

\section{Données biométriques}

Pour chaque campagne de mesure, la moyenne arithmétique et l'écart type des longueurs et des poids ont été calculés pour le lot de smolts anciennement biopsiés et le lot de 50 témoins. Les différences éventuelles ont été testées par analyse de variance et analyse de moyenne.

\section{Données physiologiques}

Pour certains échantillons de petite taille, tout ou partie des résultats de la lecture étaient négatifs : la quantité de phosphate inorganique libérée par l'activité des enzymes ATPasiques et/ou la quantité de protéines membranaires étaient inférieures au seuil de sensibilité de la méthode. Ces échantillons n'ont donc pas pu être analysés. Les autres données ont été traitées de la même manière que celles obtenues par le macrodosage.

L'équation des courbes standards était de la forme $a+b X+c \operatorname{Exp}\left(d X^{2}\right)$ pour le dosage du phosphate inorganique et $a+b x+c x^{2}$ pour le dosage des protéines. L'écart entre les duplicats était inférieur ou égal à $10 \mu \mathrm{mol}$ pour le phosphate inorganique et $2 \mu \mathrm{g}$ pour les protéines. La valeur moyenne obtenue pour chaque échantillon a donc pu être retenue. Le calcul de l'activité totale et de l'activité en présence d'ouabaïne a été effectué à partir de la formule précédemment citée. L'activité $\mathrm{Na}+, \mathrm{K}+$, ATPasique est déduite par différence entre l'activité ATPasique totale et l'activité ATPasique en présence d'ouabaïne, inhibiteur spécifique de la $\mathrm{Na}+\mathrm{K}+$ ATPase. Pour chaque lot de 12 smolts biopsiés à une même date, la moyenne arithmétique des activités $\mathrm{Na}+\mathrm{K}+$ ATPasiques, l'écart type et le coefficient de variation ont été calculés. Les différences éventuelles ont été testées par analyse de variance et de moyenne.

\section{RÉSULTATS}

\section{Survie, croissance et état sanitaire}

\section{Survie et état sanitaire}

Aucune mortalité n'a été observée à la suite des biopsies et les smolts manipulés ont montré un bon état de santé générale pendant toute la durée de l'expérimentation. Signalons, toutefois, qu'un poisson biopsié une seconde fois lors de la dernière campagne de prélèvements présentait encore des lésions sur l'arc branchial anciennement manipulé.

\section{Croissance}

Les poissons du lot biopsié n'ont pas présenté de différence significative de croissance tant linéaire que pondérale avec le lot témoin (Fig. 1 et 2, Tableau I) pendant la durée de l'étude.

\section{Analyses physiologiques}

\section{La cinétique enzymatique}

Quel que soit le dosage utilisé, l'activité $\mathrm{Na}+, \mathrm{K}+$, ATPasique augmente régulièrement au cours de la période de smoltification pour atteindre un maximum le 20 avril, puis elle décroît rapidement. Pour le microdosage, on observe cependant une remontée de l'activité à la dernière campagne de prélèvements (tableau II et fig. 3). Mais cette remontée semblerait reliée à un artefact de dosage. 


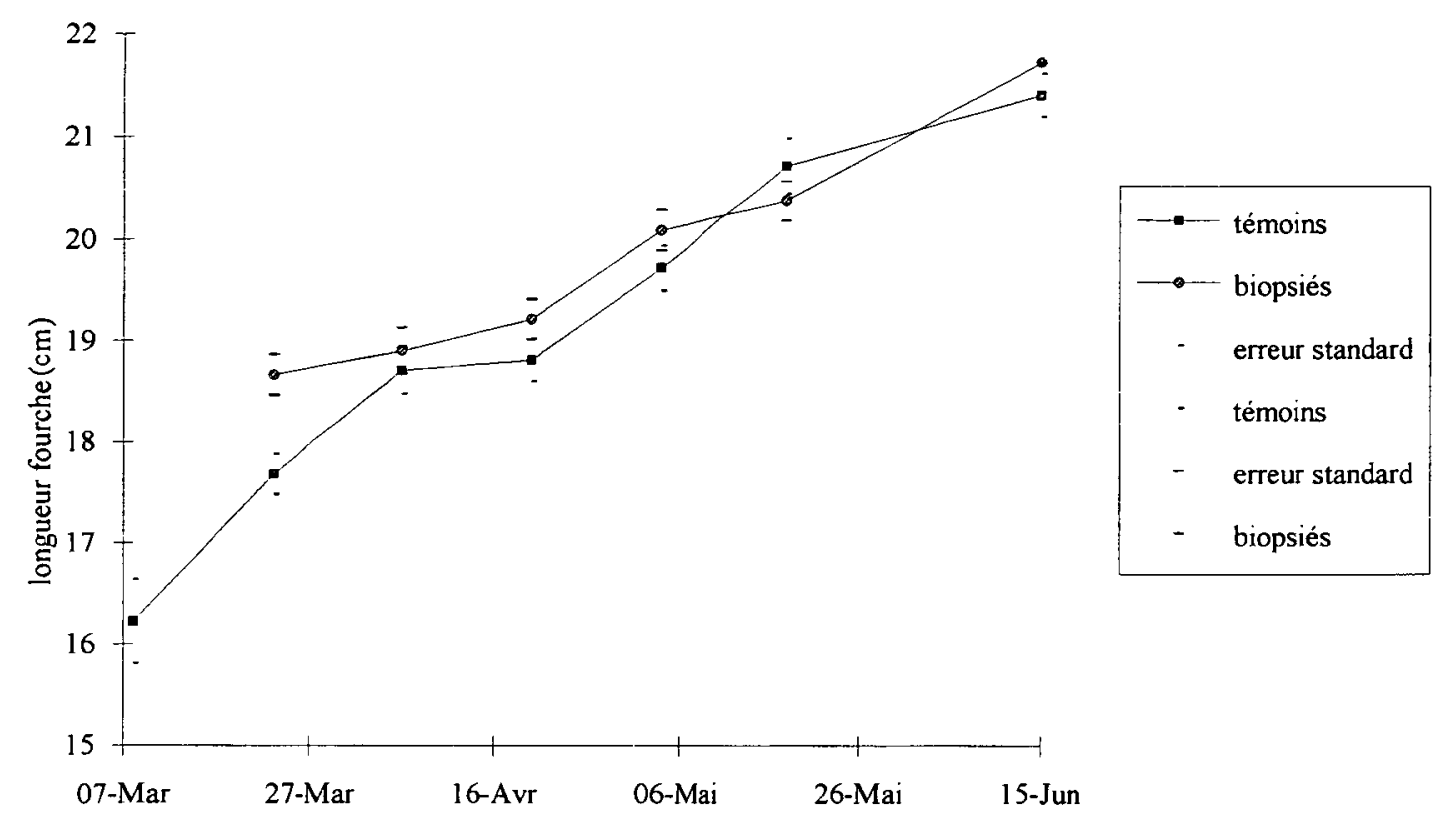

Figure 1 : Evolution dans le temps de la longueur fourche moyenne des lots témoins et biopsiés.

Figure 1 : Evolution in time of the mean fork length of the control and the gill biopsy samples.

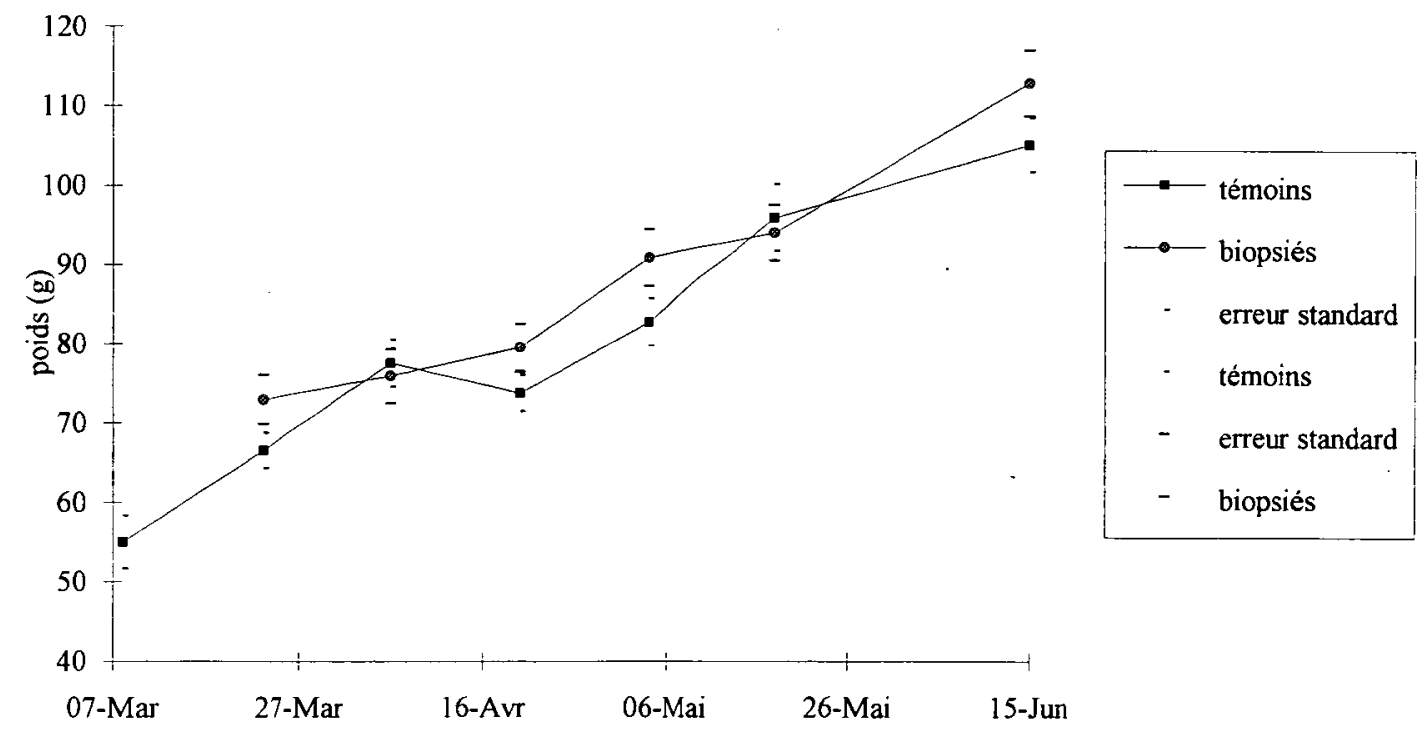

Figure 2 : Evolution dans le temps du poids moyen des lots témoins et biopsiés.

Figure 2 : Evolution in time of the mean weight of the control and the gill biopsy samples. 
Tableau I : Evolution de la taille (longueur fourche Lf en $\mathrm{cm}$ ) et du poids (P en grammes) des lots biopsiés et témoins de juvéniles de saumons Atlantique (Salmo salar) pendant la période d'étude. SE représente l'erreur standard : $\sigma / \sqrt{ }$.

Table I : Evolution of size (fork length $\mathrm{Lf}$ in $\mathrm{cm}$ ) and of weight (P in grams) of the gill biopsy and the control samples of juvenile Atlantic salmon (Salmo salar) during the duration of the study. SE is the standard error : $\sigma / \mathrm{N}$.

\begin{tabular}{|c|c|c|c|c|c|c|}
\cline { 2 - 7 } \multicolumn{1}{c|}{} & \multicolumn{4}{c|}{ lot témoin } & \multicolumn{3}{c|}{ poissons précédemment biopsiés } \\
\hline date & nombre & $\mathrm{P}+/$-SE & Lf +/-SE & nombre & $\mathrm{P}+/$ - SE & Lf +/- SE \\
\hline 8-mars & 50 & $54.96+/-3.31$ & $16.22+/-0.41$ & $/$ & $/$ & $/$ \\
23-mars & 50 & $66.5+/-2.20$ & $17.68+/-0.20$ & 12 & $72.92+/-3.06$ & $18.66+/-0.30$ \\
6-avril & 50 & $77.5+/-2.99$ & $18.7+/-0.23$ & 24 & $75.88+/-3.42$ & $18.9+/-0.2$ \\
20-avril & 50 & $73.74+/-2.28$ & $18.8+/-0.21$ & 36 & $79.5+/-3.04$ & $19.2+/-0.22$ \\
4-mai & 50 & $82.78+/-3$ & $19.7+/-0.22$ & 48 & $90.92+/-3.57$ & $20.08+/-2$ \\
18-mai & 50 & $95.96+/-4.19$ & $20.7+/-0.27$ & 60 & $94.07+/-3.5$ & $20.36+/-0.2$ \\
15-juin & 50 & $105.12+/-3.41$ & $21.4+/-0.21$ & 72 & $112.88+/-4.2$ & $21.72+/-0.19$ \\
\hline
\end{tabular}

Tableau II : Evolution dans le temps de la valeur de l'activité de la Na+/K+ ATPase branchiale mesurée par microdosage sur les lots biopsiés et par macrodosage sur des lots sacrifiés de saumons Atlantique (Salmo salar). CV représente le coefficiént de variation : $\sigma /$ moyenne.

Table II : Evolution in the time of the gill Na+/K+ ATPase activity value measured by microassay on the gill biopsy samples and by macroassay on the killed samples of Atlantic salmon (Salmo salar). CV is the coefficient of variation : $\sigma /$ mean.

\begin{tabular}{|c|c|c|c|c|c|c|c|c|}
\hline \multirow[b]{2}{*}{ date } & \multicolumn{2}{|c|}{$\begin{array}{c}\text { microdosage } \\
\text { (smolts biopsiés) } \\
\text { manipulateur } 1 \\
\end{array}$} & \multicolumn{2}{|c|}{$\begin{array}{c}\text { macrodosage } \\
\text { (smolts biopsiés) } \\
\text { manipulateur } 1\end{array}$} & \multicolumn{2}{|c|}{$\begin{array}{l}\text { macrodosage } \\
\text { (témoins) } \\
\text { manipulateur 2 }\end{array}$} & \multicolumn{2}{|c|}{$\begin{array}{c}\text { microdosage } \\
\text { (deuxième biopsie) } \\
\text { manipulateur } 1\end{array}$} \\
\hline & $\begin{array}{c}\text { nombre } \\
\text { d'individus }\end{array}$ & $\begin{array}{c}\text { activité } \\
\text { (umol/mg/h) }\end{array}$ & $\begin{array}{c}\text { nombre } \\
\text { d'individus }\end{array}$ & $\begin{array}{c}\text { activité } \\
(\mu \mathrm{mol} / \mathrm{mg} / \mathrm{h}) \\
\end{array}$ & $\begin{array}{c}\text { nombre } \\
\text { d'individus }\end{array}$ & $\begin{array}{c}\text { activité } \\
(\mu \mathrm{mol} / \mathrm{mg} / \mathrm{h})\end{array}$ & $\begin{array}{c}\text { nombre } \\
\text { d'individus }\end{array}$ & $\begin{array}{c}\text { activité } \\
(\mu \mathrm{mol} / \mathrm{mg} / \mathrm{h})\end{array}$ \\
\hline 08-mar & 9 & $\begin{array}{c}1.6 \\
\sigma=0.72 \\
\mathrm{CV}=43.6 \%\end{array}$ & & & 10 & $\begin{array}{c}30.3 \\
\sigma=4.19 \\
C V=13.8 \%\end{array}$ & & \\
\hline 23-mar & 10 & $\begin{array}{c}5.6 \\
\sigma=2.01 \\
C V=35.9 \%\end{array}$ & & & 12 & $\begin{array}{c}11.7 \\
\sigma=3.48 \\
C V=8.3 \%\end{array}$ & & \\
\hline 06-avr & 7 & $\begin{array}{c}9.6 \\
\sigma=3.37 \\
\operatorname{CV} 35.2 \%\end{array}$ & & & 12 & $\begin{array}{c}42.2 \\
\sigma=4.72 \\
C V=11.2 \%\end{array}$ & & \\
\hline 20 -avr & 12 & $\begin{array}{c}11.4 \\
\sigma=3.52 \\
C V=30.9 \%\end{array}$ & & & 11 & $\begin{array}{c}57.3 \\
\sigma=7.01 \\
\mathrm{CV}=12.2 \%\end{array}$ & & \\
\hline 04-mai & 8 & $\begin{array}{c}4.9 \\
\sigma=1.9 \\
C V=38.3 \%\end{array}$ & & & 12 & $\begin{array}{c}47.4 \\
\sigma=6.91 \\
C V=14.6 \%\end{array}$ & & \\
\hline 18-mai & 9 & $\begin{array}{c}1.8 \\
\sigma=0.68 \\
C V=38.5 \%\end{array}$ & & & 8 & $\begin{array}{c}44.4 \\
\sigma=4.34 \\
C V=9.8 \%\end{array}$ & & \\
\hline 15-jun & 12 & $\begin{array}{c}3.8 \\
\sigma=1.43 \\
\mathrm{CV}=37.2 \%\end{array}$ & 12 & $\begin{array}{c}14.626 \\
\sigma=3.42 \\
C V=23.3 \%\end{array}$ & 12 & $\begin{array}{c}19.3 \\
\sigma=2.77 \\
\mathrm{CV}=14.4 \%\end{array}$ & 11 & $\begin{array}{c}3.9 \\
\sigma=1.53 \\
C V=38.6 \%\end{array}$ \\
\hline
\end{tabular}




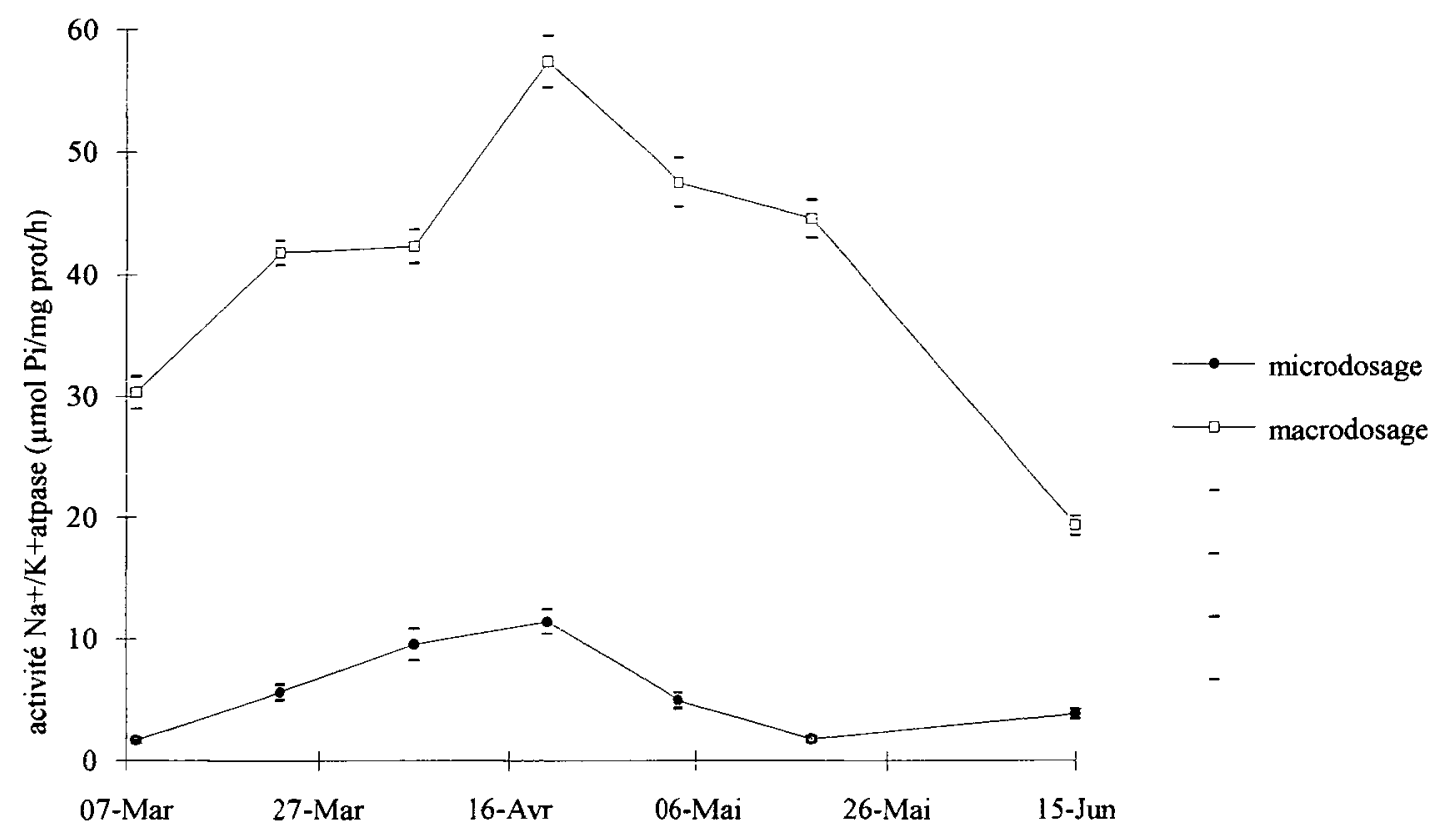

Figure 3 : Cinétique de l'activité $\mathrm{Na}+, \mathrm{K}+\mathrm{ATPasique}$ branchiale mesurée par micro et macrodosage.

Figure 3 : Kinetics of gill $\mathrm{Na}+, \mathrm{K}+\mathrm{ATPase}$ activity measured by micro and macroassay.

Les valeurs s'échelonnent entre 1.6 et $11.4 \mu \mathrm{mol} / \mathrm{mg}$ de protéines pour le microdosage et de 19 à $60 \mu \mathrm{mol} / \mathrm{mg}$ pour le macrodosage. Cependant, les coefficients de variation pour les différents lots de smolts biopsiés sont très élevés (de 30.9 à $43.6 \%$ ) pour le microdosage par rapport à ceux du macrodosage (de 9.8 à $14.6 \%$ ) et les écarts les plus importants s'observent pour les échantillons de petites tailles.

\section{Impact de la biopsie sur le développement physiologique du poisson}

Aucune différence significative n'est observée entre l'activité des smolts biopsiés une première fois le 15 juin et celle des smolts biopsiés pour la seconde fois à cette période (Tableau II). Les smolts biopsiés semblent donc poursuivre un développement physiologique similaire à celui de smolts non manipulés. biopsié

Valeur de la $\mathrm{Na}+/ \mathrm{K}+$ ATPase sur les différents arcs branchiaux d'un même poisson

II existe une grande variabilité d'activité $\mathrm{Na}+\mathrm{K}+$ ATPasique entre les différents arcs branchiaux d'un même côté et entre les arcs branchiaux de deux côtés opposés d'un même poisson (Tableau III).

\section{Comparaison entre micro et macrodosage}

La comparaison entre le micro et le macrodosage n'a été effectuée qu'à la dernière campagne de mesure où 11 smolts ont subi une biopsie puis un prélèvement total des branchies en vue d'un dosage classique (Tableau IV). Cette comparaison montre une très bonne corrélation entre les deux dosages (Fig.4) $\left(R^{2}=0.98\right.$ renforcée par la valeur du $F$ de Fisher très supérieure à la valeur critique de $F(501.2$ contre 5.12$))$. Le rapport entre macro et microdosage donne une valeur de 3.95 (Tableau IV). 
Tableau III : Activité $\mathrm{Na}+\mathrm{K}+$ ATPasique des différents arcs branchiaux d'un même poisson mesurée par microdosage au 15 juin 1994.

Table III : Na+/K+ ATPasique activity in the different gill arches of the same fish measured by microassay on the 15th of june 1994 .

\begin{tabular}{|c|c|c|}
\cline { 2 - 3 } \multicolumn{1}{c|}{} & \multicolumn{2}{c|}{ Activité $(\mu \mathrm{g} \mathrm{Pi} / \mathrm{mg}$ protéine/h) } \\
\hline arc branchial & Gauche & Droite \\
\hline 1 & 3.3 & 4.3 \\
\hline 2 & 9.5 & 8.6 \\
\hline 3 & 11.9 & 9.8 \\
\hline 4 & 6.2 & 3.4 \\
\hline
\end{tabular}

Tableau IV : Comparaison des rapports macrodosage sur microdosage. En gras italique, les valeurs trouvées par McCORMICK en 1993. CV représente le coefficient de variation : $\sigma /$ moyenne.

Table IV : Comparison of macro - microassay ratios. In heavy faced italics, the values found by McCORMICK in 1993. CV is the coefficient of variation : $\sigma /$ mean.

\begin{tabular}{|c|c|c|c|}
\cline { 2 - 4 } \multicolumn{1}{c|}{$\begin{array}{c}\text { Activité microdosage } \\
(\mu \mathrm{g} \text { Pi/mg protéine/h) }\end{array}$} & $\begin{array}{c}\text { Activité macrodosage } \\
(\mu \mathrm{g} \text { Pi/mg protéine/h) }\end{array}$ & Rapport macro/micro \\
\hline 3.7 & 13.7 & 3.69 \\
1.8 & 9.7 & 5.43 \\
2.9 & 12.8 & 4.40 \\
6.4 & 19.9 & 3.12 \\
& 2.9 & 12.9 & 4.51 \\
1.9 & 9.7 & 5.03 \\
& 4.2 & 15.1 & 3.59 \\
& 5.2 & 18.2 & 3.49 \\
& 4.1 & 14.6 & 3.59 \\
& 4.5 & 15.5 & 3.43 \\
& 6.0 & 18.9 & 3.14 \\
\hline moyenne & $4.0(3.7+/-0.5)$ & $14.6(16+/-1)$ & $3.7(4.3)$ \\
$\sigma$ & 1.527 & 3.406 & 0.78 \\
CV $(\%)$ & 38.53 & 23.29 & 19.66 \\
\hline
\end{tabular}

\section{DISCUSSION}

L'expérimentation réalisée ici a permis de confirmer à moyen terme (contrôle continu sur presque quatre mois), les observations de MCCORMICK (1993) en milieu contrôlé et de SHROCK et al. (1994) en milieu naturel concernant la croissance et la survie des poissons. Les poissons biopsiés ne présentent pas de différences significatives de croissance linéaire et pondérale avec les poissons non manipulés et la survie n'a été aucunement diminuée, et ce, quelle que soit la date de prélèvement durant la période mars à juin et le niveau de smoltification des poissons. De plus, il apparaît que la biopsie ne semble pas altérer le 


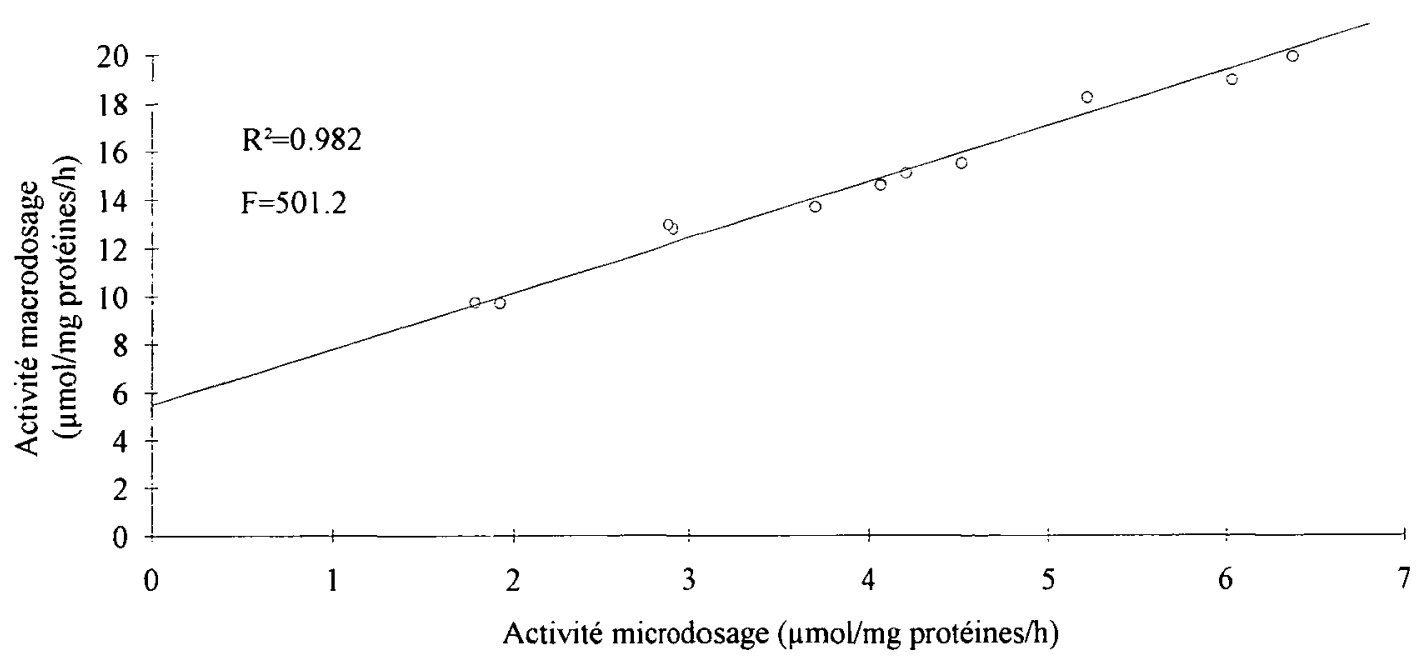

Figure 4 : Relation entre microdosage et macrodosage au 15 juin 1994.

Figure 4 : Relationship between microassay and macroassay on the 15th of june 1994.

développement physiologique ultérieur du poisson puisque les niveaux d'activité $\mathrm{NA}+\mathrm{K}+$ ATPasiques ne sont pas différents en fin d'expérimentation pour les poissons biopsiés pour la deuxième fois que pour les poissons manipulés pour la première fois. Cette observation mériterait toutefois d'être confirmée par l'analyse de la cinétique afin de préciser si la montée et le maximum d'activité $\mathrm{Na}+\mathrm{K}+$ ATPasique ne sont pas modifiés. Néanmoins, ces premières observations permettent de montrer que la biopsie n'affecte pas le smolt dans sa croissance ni dans sa physiologie. De plus, la cicatrisation semble être rapide puisque sur toute la période d'étude, seul un poisson a présenté une plaie ouverte au contrôle suivant.

Les valeurs d'activité que nous avons obtenues par microdosage sont du même ordre que celles obtenues par McCORMICK (1993). La courbe traçant l'évolution de l'activité $\mathrm{Na}+/ \mathrm{K}+$ ATPasique dans le temps est également conforme à ce que l'on pouvait attendre dans pareil cas, si on excepte le dernier point de mesure qui fait remonter l'activité. La courbe d'activité obtenue par macrodosage confirme d'ailleurs l'allure générale de la courbe de microdosage et la date du pic d'activité (fig.3). Toutefois, macro et microdosages n'ont pas été effectués sur les mêmes poissons et les différences de profil des deux courbes obtenues ne peuvent donc pas être imputées plus à la différence de méthode utilisée qu'à la variabilité interindividuelle (JOHNSON et al., 1977). Cette observation est renforcée par la très bonne corrélation que l'on retrouve entre micro et macrodosages effectués sur les mêmes poissons à la dernière campage de mesures (fig.4). Par ailleurs, la corrélation obtenue a une valeur $(r=0.991)$ supérieure à celle obtenue en moyenne par SCHROCK et al. (1994) $(r=0.96)$. De plus, le rapport entre l'activité mesurée par macrodosage et l'activité mesurée par microdosage (Tableau IV) est du même ordre de valeur que les 4.3 obtenus pour des présmolts et les 3.95 obtenus pour des smolts par MCCORMICK (1993) et permet de lever le doute. II serait maintenant intéressant de déterminer si cette corrélation se confirme à tout moment de la cinétique et s'il existe une relation stable entre micro et macrodosage quelle que soit la période du prélèvement.

Cependant, la technique de microdosage mise au point pour cette expérimentation donne des coefficients de variation allant de 30.9 à $43.6 \%$, ce qui est élevé en 
comparaison avec les $12 \%$ de moyenne obtenus par macrodosage. De tels écarts deviennent rédhibitoires pour une comparaison de cinétique enzymatique selon l'année et le site géographique chez le saumon et pour une analyse d'activité chez la truite fario (Salmo trutta L.) où les variations inter-individus sont déjà très importantes (SIEGLER, données non publiées). II y a sans doute une responsabilité non négligeable de l'opérateur dans la fluctuation obtenue. Sur deux dosages similaires (macrodosages du 15 juin), deux manipulateurs différents ont obtenu des coefficients de variation de 23.3 et $14.4 \%$ respectivement (Tableau II). On peut donc aisément imaginer qu'une différence sensible se serait retrouvée lors d'un microdosage. Il est donc préférable que les dosages soient effectués par un même expérimentateur entraîné afin d'aboutir à une meilleure homogénéité des résultats. Néanmoins, même si cette variabilité est du même ordre de grandeur que celle calculée à partir des résultats de McCORMICK (1993), soit $33.7 \%$, le protocole de prélèvement ou de dosage doit pouvoir être affiné. Quelques améliorations peuvent d'ores et déjà être proposées.

La technique de dosage apparaît être sensible à la quantité de tissus prélevés (fig.5), le broyage unique effectué étant sans doute plus efficace sur des gros échantillons. Ce détail technique pourrait expliquer, entre autres, la remontée surprenante d'activité lors de la dernière campagne de prélèvement où les poissons ont été sacrifiés ; le manipulateur a sans doute moins hésité à prélever un échantillon de tissu branchial plus important. II serait donc nécessaire de déterminer la taille d'échantillon maximum prélevable sans préjudice et ce, sur des poissons sauvages moins résistants aux manipulations que des poissons d'élevage (ce qui n'a jamais été fait jusqu'à présent).

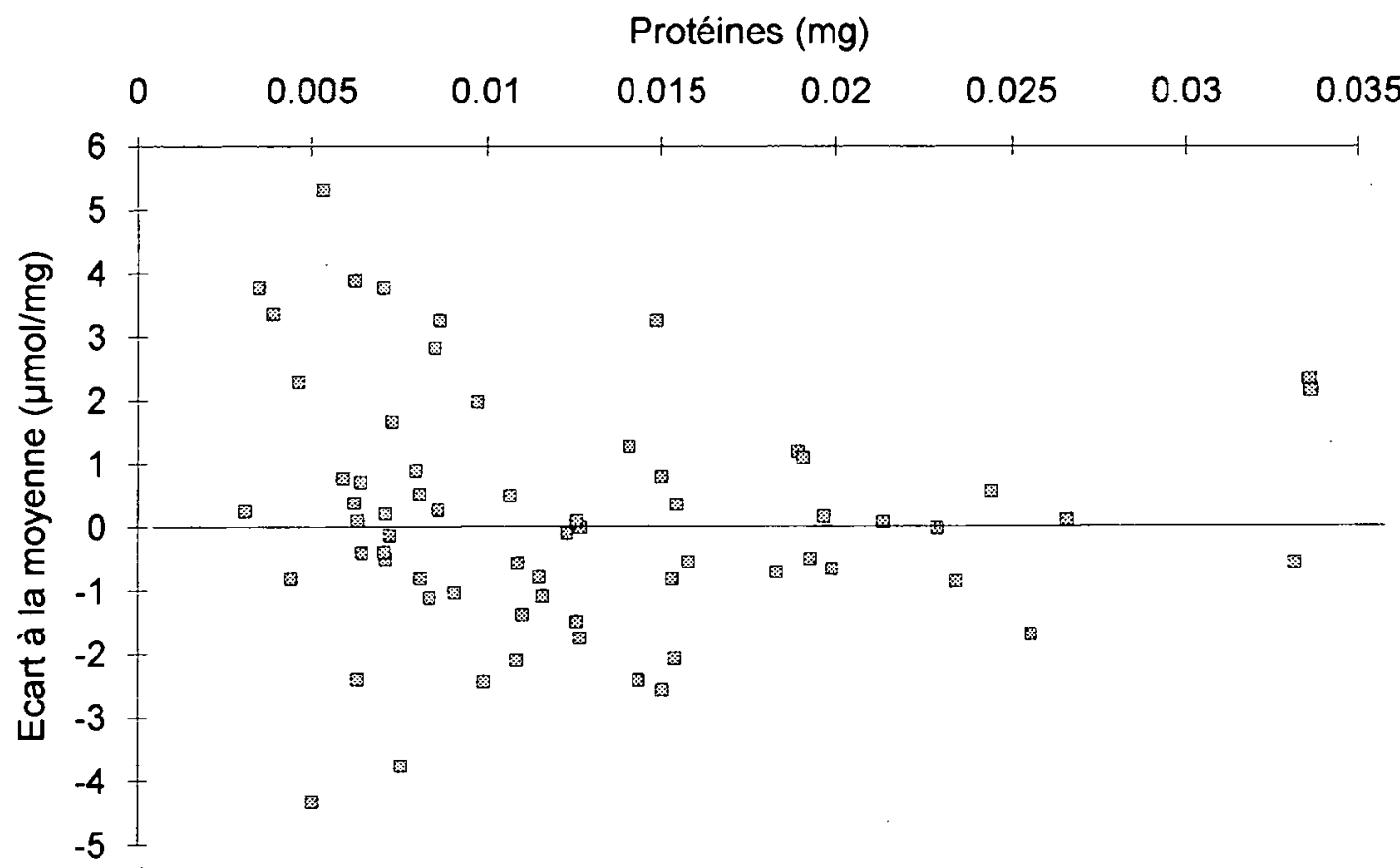

Figure 5 : Influence de la quantité de tissu branchial prélevé (exprimée en $\mathrm{mg}$ de protéines) sur la variabilité du microdosage ( $\mu$ mole de $\mathrm{Pi} / \mathrm{mg}$ de protéines).

Figure 5 : Effect of the quantity of gill tissue removed (expressed in $\mathrm{mg}$ of proteins) on the variability of the microassay ( $\mu \mathrm{mol}$ of $\mathrm{Pi} / \mathrm{mg}$ of proteins). 
De plus, il apparaît que les niveaux de $\mathrm{Na}+\mathrm{K}+\mathrm{ATPase}$ sont plus importants sur les arcs branchiaux 2 et 3 (Tableau III). II serait sans doute appréciable de standardiser le prélèvement sur un de ceux-ci, même si le prélèvement se révélait plus délicat que sur l'arc 1. En effet des niveaux d'activité plus importants sont moins assujettis à la précision du dosage (SCHROCK et al., 1994).

La méthode de biopsie branchiale est donc intéressante, telle qu'elle, pour des études ponctuelles, chez certaines espèces, lorsque l'on veut connaître le niveau d'activité $\mathrm{Na}+/ \mathrm{K}+\mathrm{ATPasique}$ d'un poisson sans le tuer. Mais, pour la même problématique chez la truite fario (Salmo trutta), ou pour faire une comparaison spatio-temporelle des niveaux $\mathrm{Na}+\mathrm{K}+\mathrm{ATPasiques}$ chez le saumon Atlantique (salmo salar), cette technique se révèle imprécise et il est nécessaire de l'améliorer. Si les modifications proposées permettent de réduire de façon significative la variabilité des résultats, la biopsie pourra alors être utilisée, en milieu naturel, pour nos études actuelles sur les différents écotypes de truites fario (Salmo trutta). Elle nous permettrait ainsi de relier le niveau de l'activité de la NA+-K+ ATPase au phénotype et au devenir du juvénile sans tuer l'individu de façon à ne pas modifier d'une manière artificielle les paramètres de la dynamique de population des deux formes migrante et résidante et permettre ainsi un suivi comparatif entre les années et les différents stocks.

Ce travail a été réalisé dans le cadre de la convention INRA-CSP $n^{\circ} 93148$ concernant la capacité adaptative de la truite (Salmo trutta L.).

\section{BIBLIOGRAPHIE}

ATKINSON A., GATENBY A.D., LOWE A.G., 1973. The determination of inorganic orthophosphate in biological systems. Biochimica and Biophysica Acta, 320, 195-204.

BOEUF G., 1992. Salmonids smolting : a pre-adaptation to the oceanic environment. In : RANKIN J.C., JENSEN F.B. (éditeurs), Fish Ecophysiology, 105-135, Chapman \& Hall, London.

BOEUF G., HARACHE Y., 1982. Criteria for adaptation of Salmonids to high salinity seawater in France. Aquaculture, 28, 163-176.

BRADFORD M. M., 1976. A rapid and sensitive method for quantization of microgram quantities of protein utilizing the principle of protein-dye binding. Anal. Biochem., 72, 374-381.

FOLMAR L.C., DICKHOFF W.W., 1980. The parr-smolt transformation and seawater adaptation in salmonids. A review of selected literature. Aquaculture, 21, 1-37.

JOHNSON S.L., EWING R.D., LICHATOWICH J.A., 1977. Characterization of gill $(\mathrm{Na}+\mathrm{K}+)$ Activated Adenosine Triphosphatase from Chinook Salmon, Oncorhynchus tschawytscha. Journal of Experimental Zoology, 199, 345-354.

LASSER P., BOEUF G., HARACHE Y., 1978. Osmotic adaptation of Oncorhynchus kisutch. I : seasonal variations of gill $\mathrm{Na}+\mathrm{K}+\mathrm{ATPase}$ activity in coho salmon, $0+$ age and yearling, reared in freshwater. Aquaculture, 14, 365-382.

McCORMICK S.D., 1993. Method for nonlethal Gill biopsie and measurement of Nat, K+ATPase activity. Canadian Journal of Fisheries and Aquatic Sciences, 50, 656-658.

McCORMICK S.D., NAIMAN R.J., MONTGOMERY E.T., 1985. Physiological smolt characteristics of anadromous and non anadromous brook trout (Salvelinus fontinalis) and Atlantic salmon (Salmo salar). Canadian Journal of Fisheries and Aquatic Sciences, 42, 529-538.

ROPARS L., 1993. Comparaison des caractéristiques éco-physiologiques des juvéniles migrants de truite commune (Salmo trutta) et de saumon Atlantique (Salmo salar) sur la rivière Oir (Basse-Normandie). DEA Biologie et Agronomie, Université de Rennes, $30 \mathrm{p}$. 
SCHROCK R.M., BEEMAN J.W., RONDORF D.W., HANER P.W., 1994. A microassay for gill sodium, potassium-Activated ATPase in Juvenile Pacific Salmonids. Transactions of the American Fisheries Society, 123, 223-229.

SOIVIO A., MUONA M., VIRTANEN E., 1989. Smolting of two populations of Salmo trutta. Aquaculture, 82, 147-153.

STAGG R.M., SHUTTLEWORTH J.J., 1982. Na+/K+ ATPase ouabain binding and ouabain sensitive oxygen consumption in gills from Platichtythys flesus adapted to seawater and freshwater. Journal of Comparative Physiology, 147, 93-99.

TANGUY J.M., 1993. La smoltification de la truite de mer (Salmo trutta) : caractérisation éco-physiologique des juvéniles en milieu contrôlé et en milieu naturel. Thèse de Doctorat, Productions Animales, ENSA Rennes, $106 \mathrm{p}$.

ZAUGG W.S., 1982. A simplified preparation for Adenosine Triphosphatase determination in gill tissue. Canadian Journal of Fisheries and Aquatic Sciences, 39, 215-217. 